infarction, anaphylactic reaction, azoospermia, liver toxicity, and severe nausea in 1 patient each. Overall, 16 malignancies were observed in $14(7 \%)$ patients after a median follow up of 25 (IQR:15-26) years. The malignancies were bladder carcinoma $(n=4)$, lung adenocarcinoma $(n=3)$, prostate adenocarcinoma $(n=2)$, carcinoma of unknown primary origin, pancreas adenocarcinoma, t-MDS-AML, lymphoma, colon adenocarcinoma, squamous cell carcinoma and thyroid papillary carcinoma. Among the 113 patients, we were able to question regarding infertility, 67 patients (59\%) had children, $22(19.5 \%)$ did not wish to have a child and $24(21.5 \%)$ tried to have a child, but was not able to.

Conclusions: Short term serious adverse events occurred in $8 \%$ of the patients during CYC treatment. During long term follow-up malignancies occurred in $7 \%$ and infertility in $21.5 \%$ of the patients. These results underline the need for safer and effective alternatives to CYC for serious organ involvement in BS, similar to that in other vasculitides.

Disclosure of Interest: None declared

DOI: 10.1136/annrheumdis-2018-eular.4817

\section{AB0704 CLINICAL-ANALYTICAL CHARACTERISATION OF 52 DIAGNOSED PATIENTS OF BEHÇET DISEASE WITH INCLUSION OF PAEDIATRIC CASES IN A SPANISH TERTIARY HOSPITAL}

\section{S. Zegarra Mondragon, A. Alia-Jimenez, C. Bouroncle Alaluna, A. Boteanu.} Rheumatology, Hospital Ramon y Cajal (Spain), Madrid, Spain

Background: Behçet's disease (BD) is a chronic and recurrent inflammatory disease of unknown etiology, classified into polygenic autoinflammatory diseases or variable vessel vasculitis. It has a wide spectrum of symptoms with a very variable range of severity, from mucocutaneous involvement to neurological manifestations, systemic vascular or severe ocular manifestations. About $5.4 \%-7.6 \%$ of Behçet's cases have a paediatric debut.

Objectives: To evaluate and compare the clinical and laboratory manifestations of a series of 52 patients, adults and children, diagnosed with BD according to the classification criteria of the International Study Group of BD (ISGBD-1990).

Methods: Retrospective cross-sectional observational study, which included 43 adult patients and 9 paediatric patients diagnosed with EB in the Rheumatology Department of a Madrid tertiary hospital. The clinical-analytical characteristics of both groups were evaluated, as well as the correlation of HLA-B51 with the described symptomatology.

Results: The mean age at diagnosis of BD was $36.9 \pm 11.8$ years in adults and $11.4 \pm 5.1$ years in children. $27.3 \%$ of adults and $11.1 \%$ of children with BD were male, with oral ulcers close to $90 \%$ in both groups. Contrary to what was reported in other series, genital ulcers were more frequent in children $(77.8 \%$ versus $65.9 \%$ of adults), as was the presence of uveitis (44.4\% in children compared to $22.7 \%$ in adults) and neurological manifestations $(22.2 \%$ in children versus $6.8 \%$ in adults). Joint involvement was also more frequent in children $(88.9 \%$ versus $52.3 \%$ in adults), as well as fever ( $44 \%$ in children versus $14 \%$ in adults); being these two manifestations the only parameters that were associated in a statistically significant way with their presentation in the paediatric age in BD. In contrast, skin involvement and vascular manifestations were more frequent in adults. The positivity of HLA-B51 did not correlate statistically with any clinical manifestation, but those who had it had a mean age at diagnosis of 26.5 years compared to a mean of 39 years in those who did not present this genetic marker.

Conclusions: Behçet's disease presents with a wide spectrum of clinical manifestations, potentially serious, ranging from skin lesions to neurological or vascular manifestations. In our series, patients diagnosed at paediatric age most frequently had systemic manifestations (fever), arthritis or severe clinical manifestations such as neurological involvement or uveitis. Limitations: a small number of paediatric cases included in our study.

Disclosure of Interest: None declared

DOI: 10.1136/annrheumdis-2018-eular.6188

\section{AB0705 LONG-TERM OUTCOMES AND PROGNOSTIC FACTORS ASSOCIATED WITH AORTIC VALVE SURGERY IN PATIENTS WITH TAKAYASU ARTERITIS AND AORTIC VALVEREGURGITATION}

S.H. Nam ${ }^{1}$, O.C. Kwon ${ }^{1}$, W.J. Seo ${ }^{2}$, Y.-G. Kim ${ }^{1}$, C.-K. Lee ${ }^{1}$, B. Yoo ${ }^{1}$, S. Hong ${ }^{1}$ ${ }^{1}$ Internal medicine, Asan Medical Center, ${ }^{2}$ Internal medicine, Veterans Health Service Medical Center, Seoul, Korea, Republic of Ireland

Background: Some patients with Takayasu arteritis (TA) have aortic valve (AV) involvement, which can lead to aortic regurgitation (AR). However, data on the long-term outcomes, including survival of TA patients with AR, are lacking. Moreover, previous studies were limited to patients who underwent AV surgery.
Objectives: This study aimed to characterise the long-term outcomes and clinical characteristics of TA patients with AR regardless of whether they underwent surgical intervention.

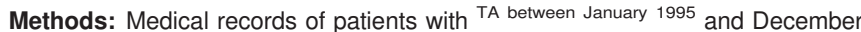
2015 were retrospectively reviewed. AR was diagnosed using transthoracic echocardiography. Poor outcomes were defined as all-cause death and major adverse cardiac and cerebrovascular events (MACCE). Multivariate analysis was per formed to determine the factors affecting poor prognosis in the surgical group.

Results: Of the total 105 patients with TA and AR, 41 (39.0\%) underwent AV surgery. Among patients who underwent AV surgery, inflammation values (Erythro cyte sedimentation rate, $62.07 \pm 31.8 \mathrm{~mm} / \mathrm{hr}$ vs. $39.16 \pm 28.4 \mathrm{~mm} / \mathrm{hr}$; C-reactive protein, $3.66 \pm 4.1 \mathrm{mg} / \mathrm{dL}$ vs. $0.92 \pm 1.7 \mathrm{mg} / \mathrm{dL})$, AR degree $(3.56 \pm 0.7$ grade vs $2.08 \pm 1.0$ grade), and sinus diameter $(37.24 \pm 5.7 \mathrm{~mm}$ vs. $33.22 \pm 4.5 \mathrm{~mm})$ were significantly higher than in those who did not undergo AV surgery. Long-term survival and freedom from MACCE were not significantly different between the groups (10 year survival, $84.3 \%$ vs. $79.4 \% ; p=0.827 ; 10$ year event-free survival, $51.8 \%$ vs. $71.2 \% ; p=0.29$ ). Twelve of the 41 patients who underwent AV surgery had a poor outcome during follow-up (median, 92.5 months; IQR, 54.5-183.5), and eight of them had a recurrence of AR requiring reoperation. Multivariate Cox analysis revealed that coronary disease [hazard ratio $(\mathrm{HR}), 4.234 ; 95 \%$ confidence interval $(\mathrm{Cl}), 1.381-12.979 ; \mathrm{p}=0.012$ ], LV dysfunction $(\mathrm{HR}, 3.387 ; 95 \% \mathrm{Cl}$ 1.143-10.042; $p=0.028$ ), and impaired renal function (HR, 19.983; 95\% Cl, $3.480-114.731 ; p=0.001)$ were significant risk factors associated with poor outcomes at follow-up (table 1).

Abstract AB0705 - Table 1. Multivariate analysis of predictive factors of poor outcomes in patients with Takayasu arteritis who underwent aortic valve surgery.

\begin{tabular}{lccc}
\hline Variables & $\mathrm{HR}$ & $95 \% \mathrm{Cl}$ & $\begin{array}{c}\mathrm{p}- \\
\text { value }\end{array}$ \\
\hline Coronary disease $^{*}$ & 4.234 & $1.381-12.979$ & 0.012 \\
LV dysfunction** $^{*}$ & 3.387 & $1.143-10.042$ & 0.028 \\
Impaired renal & 19.983 & $3.480-$ & 0.001 \\
function & & 114.731 &
\end{tabular}

*coronary disease: severity is more than moderate stenosis

${ }^{* *}$ LV dysfunction: $\mathrm{EF}<50 \%$

Conclusions: In patients with TA with AV involvement, there were no significant differences between long-term survival rate and event-free survival (MACCE) among those who had or had not undergone AV surgery. In the surgical group, the prognosis was poor when coronary artery disease, LV dysfunction, and rena impairment were present at the time of surgery.

Disclosure of Interest: None declared

DOI: 10.1136/annrheumdis-2018-eular.3042

\section{AB0706 OCULAR PRESENTATION IN GRANULOMATOSIS WITH POLYANGIITIS (GPA) PATIENTS: RELATION TO AUTOANTIBODIES AND DISEASE ACTIVITY}

T.A. Gheita ${ }^{1}$, E.M. Abd El-Latif ${ }^{2} .{ }^{1}$ Rheumatology and Clinical Immunology, Faculty of Medicine, Kasr Al-Ainy School of Medicine, Cairo University, Cairo;

${ }^{2}$ Ophthalmology, Faculty of Medicine, Alexandria University, Alexandria, Egypt

Objectives: To study the disease characteristics, autoantibodies and activity in granulomatosis with polyangiitis (GPA) patients with ocular manifestations.

Methods: 46 GPA patients visiting the ophthalmology clinic were included. Ocular manifestations, clinical and slit lamp examination were performed. The Birmingham Vasculitis Activity Score (BVAS) was recorded. Laboratory investigations were recorded and the antineutrophil cytoplasmic antibody (ANCA) performed.

Results: The median age of the patients was 44.5 (32-63) years, 22 males:24 females and disease duration 6.5 (1-16) years. Ocular manifestations were present in all patients; $12(26.1 \%)$ had proptosis, $40(87 \%)$ scleritis/episcleritis with perforation in $3(6.5 \%)$, keratoconjunctivitis in $33(71.7 \%)$ - acute infiltrative stromal keratitis in 11, peripheral ulcerative keratitis in 15 and sclerosing keratitis in 11 patients. Uveitis was present in 11 (23.9\%) and retinal changes included vasculitis, exudates and haemorrhage was present in $7(15.2 \%) .43(93.5 \%$ of the patients had blurring of vision and vision loss was present in $2(4.3 \%)$. Glaucoma was present in $4(8.7 \%)$ and hypotony in $2(4.3 \%)$. Involvement was bilateral in 32 $(69.6 \%)$ patients. Rheumatoid factor was positive in $56.5 \%$ and significantly associated with uveitis $(p=0.04)$ while ANA was positive in $45.7 \%$ and significantly associated with keratoconjuctivitis $(p=0.04)$. BVAS tended to be higher in those with uveitis $(p=0.05)$.

Conclusions: Ocular involvement must be considered in all GPA patients and referral to an experienced ophthalmologist is mandatory for proper management and improved outcome of such a rare systemic disease. ANA and RF positivity may raise suspicion for $\mathrm{KC}$ or uveitis respectively. There was a remarkable association between uveitis and disease activity. 
Disclosure of Interest: None declared

DOI: 10.1136/annrheumdis-2018-eular.5230

\section{AB0707 1 FOUR DISTINCT CLINICAL PHENOTYPES OF VASCULITIS AFFECTING MEDIUM-SIZED ARTERIES}

T. Shirai, H. Fujii, Y. Shirota, T. Ishii, H. Harigae. Hematology and Rheumatology, Tohoku University Graduate School of Medicine, Sendai, Japan

Background: Polyarteritis nodosa (PAN) is a necrotizing arteritis of mediumsized arteries. PAN is divided into systemic and cutaneous PAN (cPAN). cPAN is further classified into mild cPAN or severe cPAN, which presents with ulcer, necrosis, or neuritis. However, it is sometimes difficult to distinguish between severe cPAN and systemic PAN, and their optimal managements are still unclear. The aim of this study is to evaluate clinical characteristics of patients with necrotizing arteritis of medium and small artery.

Objectives: To evaluate the clinical characteristics of patients with necrotizing arteritis of medium-sized arteries in order to further clarify the subtypes of PAN. Methods: Forty-nine patients diagnosed with necrotizing arteritis of mediumsized arteries between 2008 and 2017 at our institution were enrolled to this study Patients with evidence of glomerulonephritis or vasculitis in arterioles, capillaries, or venules, which are hallmarks of small-vessel vasculitis, were excluded. Clinical backgrounds, laboratory findings including inflammatory markers and antineutrophil cytoplasmic antibodies (ANCA), affected organs, treatments, and rates of relapse and death were evaluated.

Results: Among 49 patients, 11 patients (22\%) presented with systemic vasculitis. Organ involvement was diverse and ranged from central nervous system to cutaneous manifestations. The remaining 38 patients were diagnosed as cPAN and further classified as mild CPAN (16 patients) or severe CPAN (22 patients: ulcer type, 9; neuritis type, 9; both, 4). There was one case of cPAN which resulted in renal damage. The clinical characteristics of mild cPAN included female predominance (87.5\%) and younger age (median, 32), and patients tend to have normal inflammatory markers and laboratory findings. Those of systemic PAN included older age (median, 69), higher levels of inflammatory markers, lower levels of serum proteins, and organ damage. Nonspecific elevation of autoantibodies is frequently observed. Particulary, $54.5 \%$ of systemic type possessed MPOANCA though the titers were significantly lower than those of microscopic polyangiitis, suggesting non-specific elevation of MPO-ANCA in Japanese population. Severe cPAN manifested with intermediate phenotypes, and inflammatory activities were significantly correlated with age $(p<0.001)$. Although the mortality rates were indistinguishable, the relapse rates of cPAN (ulcer type) were significantly higher than those of other types (88.9\%, Figure). The mean doses of prednisolone (PSL) used to treat mild cPAN, severe cPAN, and systemic type were 18.5, 38.6, and $39.1 \mathrm{mg} /$ day, respectively. Immunosuppressants were used in $20 \%$ of mild cPAN, $90.9 \%$ of severe cPAN, and $72.7 \%$ of systemic PAN patients. Most patients with cPAN (ulcer type) were initially treated by corticosteroid monotherapy, and immunosuppressive agents were added when they relapsed. Considering its high relapse rate, it might be reasonable to select combination therapy with cyclophosphamide for severe cPAN (ulcer type) as is proposed in nonsystemic vasculitic neuropathy.

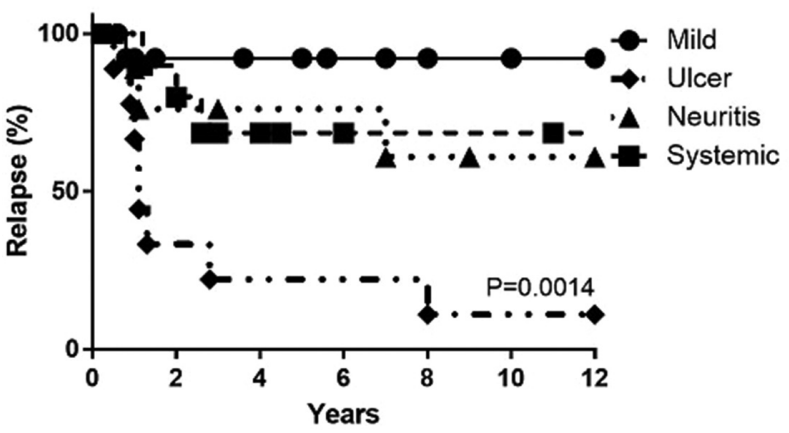

$\begin{array}{cccccccc}\text { Number at risk } & 0 y & 2 y & 4 y & 6 y & 8 y & 10 y & 12 y \\ \text { Mild } & 16 & 15 & 11 & 9 & 7 & 4 & 2 \\ \text { Ulcer } & 9 & 3 & 2 & 2 & 1 & 1 & 1 \\ \text { Neuritis } & 9 & 7 & 5 & 5 & 2 & 1 & 1 \\ \text { Systemic } & 11 & 9 & 7 & 5 & 2 & 2 & 1\end{array}$

Abstract AB0707 - Figure 1
Conclusions: The clinical characteristics of mild cPAN, severe cPAN (ulcer type), severe cPAN (neuritis type), and systemic PAN were distinct from each other. Particularly, patients with severe cPAN (ulcer type) had higher relapse rates, thus indicating the importance of combination therapy in this patient cohort. Disclosure of Interest: None declared

DOI: 10.1136/annrheumdis-2018-eular.2247

\section{AB0708 DIAGNOSIS AND MANAGEMENT OF PATIENTS WITH GIANT CELL ARTERITIS (GCA)- A RE-AUDIT IN RHEUMATOLOGY IN THE BELFAST HEALTH AND SOCIAL CARE TRUST (BHSCT)}

\section{U.A. Laverty, E. Banks, M. McHenry. NHS, Belfast, Ireland}

Background: Patients should meet at least 3/5 American College of Rheumatology (ACR) criteria for a diagnosis of GCA. British Society of Rheumatology (BSR) guidelines on the management of GCA from 2010 emphasise the importance of early referral for specialist management. TAB should be considered depending on local experience and availability of other imaging modalities. Biopsy should be at least $10 \mathrm{~mm}$ in length and ideally done within 14 days of presentation. TABs performed in BHSCT over a three-year period were audited in 2014.

Objectives: We re-audited the Rheumatology TAB referrals in the BHSCT from August 2016 to August 2017 to assess how biopsies influenced the management of patients with GCA in comparison to the previous audit.

Methods: Histopathology based LabCentre search detected 36 TABs within the given period. A retrospective audit was carried out with the use of Electronic Care Record and 16 of these patients were Rheumatology based TAB referrals. A proforma was used to aid data collection.

Results: Female:Male ratio was 2.2:1, age range was 61-91 with a mean age of 76. $94 \%$ of patients presented with headache. Of those patients referred for biopsy, $100 \%$ already fulfilled 2 of ACR criteria, compared with only $80 \%$ in previous audit. TAB was positive in $25 \%$. In those with a positive biopsy, $75 \%$ had an ESR $>50$. All patients were on steroid treatment at the time of biopsy. In $42 \%$ of patients with a negative biopsy, steroids were rapidly reduced. $100 \%$ of patients with a positive biopsy continued on steroids. $68 \%$ of biopsies were greater than $10 \mathrm{~mm}$ compared with $38 \%$ in the previous audit. $68 \%$ of patients had a TAB within 14 days of commencing on steroids, with $31 \%$ having biopsy within 7 days of commencing steroids. $63 \%$ of patients developed steroid related complications. In $56 \%$ of patients the biopsy result changed patient management.

Conclusions: The percentage of appropriate referrals for biopsy (based on the ACR criteria) has improved compared with the previous audit. The length of biopsy improved. $32 \%$ of patients were waiting longer than 2 weeks for biopsy but some of these patients presented late to the rheumatology team. Biopsy results changed management in $56 \%$ of patients. Areas for consideration include improvement in time to biopsy and biopsy length.

\section{REFERENCE:}

[1] BSR and BHPR guidelines for management of giant cell arteritis. Bhaskar Dasgupta, et al. Rheumatology, Volume 49, Issue 8, 1 August 2010 Pages 1594-1597. Published: 05 April 2010

Disclosure of Interest: None declared

DOI: 10.1136/annrheumdis-2018-eular.7339

\section{AB0709 1 SWITCHING FROM ORIGINATOR INFLIXIMAB TO BIOSIMILAR INFLIXIMAB: EFFICACY AND SAFETY IN A COHORT OF PATIENTS WITH ESTABLISHED BEHÇET'S DISEASE}

V. Venerito $^{1}$, G. Lopalco ${ }^{1}$, L. Cantarini ${ }^{2}$, C. Fabiani ${ }^{3}$, M. Nivuori ${ }^{1}$, F. Cacciapaglia ${ }^{1}$, M. Galeazzi ${ }^{2}$, G. Lapadula', F. lannone ${ }^{1} .{ }^{1}$ Department of Emergency and Organ Transplantations, Rheumatology Unit, University of Bari "Aldo Moro", Bari; ${ }^{2}$ Department of Medical Sciences, Surgery and Neurosciences, Rheumatology Unit, Policlinico "Le Scotte", University of Siena, Siena; ${ }^{3}$ Department of Ophthalmology, Humanitas Clinical and Research Center, Rozzano, Italy

Background: Infliximab (IFX) has been proved to be effective in several organ involvement of Behçet's Disease (BD). A recent report ${ }^{1}$ describing rapid loss of efficacy of biosimilar IFX after switching from originator IFX suggests the necessity to exercise caution regarding the automatic substitution of originator IFX with biosimilar IFX in patients achieving remission with originator IFX.

Objectives: The purpose of the present study was to describe our experience with biosimilar IFX CT-P13 in patients affected with BD, who were switched from originator IFX. 